\title{
Noun Countability; Count Nouns and Non-count Nouns, What are the Syntactic Differences Between them?
}

\author{
Azhar A. Alkazwini \\ The Public Authority of Applied Education (PAAET), Kuwait \\ E-mail: aasalkb@hotmail.com
}

Received: 13-08-2016

Published: 10-12-2016
Accepted: 17-10-2016

doi:10.7575/aiac.ijalel.v.5n.7p.96
Advance Access Published: November 2016

URL: http://dx.doi.org/10.7575/aiac.ijalel.v.5n.7p.96

\begin{abstract}
Words that function as the subjects of verbs, objects of verbs or prepositions and which can have a plural form and possessive ending are known as nouns. They are described as referring to persons, places, things, states, or qualities and might also be used as an attributive modifier. In this paper, classes and subclasses of nouns shall be presented, then, noun countability branching into count and non-count nous shall be discussed. A number of present examples illustrating differences between count and non-count nouns and this includes determiner-head-co-occurrence restrictions of number, subject-verb agreement, in addition to some exceptions to this agreement rule shall be discussed. Also, the lexically inherent number in nouns and how inherently plural nouns are classified in terms of (+/- count) are illustrated. This research will discuss partitive construction of count and non-count nouns, nouns as attributive modifier and, finally, conclude with the fact that there are syntactic difference between count and non-count in the English Language.
\end{abstract}

Keywords: English Language, Nouns, Count, Non-count, Syntactic Differences, Proper Nouns

\section{Introduction}

What is meant by the Classifications of Nouns? The basic terms are not universal and are connected with different typologies of nouns. Many practical grammars mention such types of nouns in English: common, proper, abstract, collective, which are further on classified as countable (count) or uncountable (uncount, mass) (Thomson, Martinet, 1986:16; Powell 1989:5-6; Robbins 2007:2-3).

The following three properties are found in the main members of the word-class noun:

a. Functional: In the structure of NP, they function as head. Several functions are performed by NP such as subject, object or predictive complement in clause structure, and complement in PP (prepositional phrase).

b. Dependents: With nouns as head, a number of dependents occur exclusively or almost exclusively. "The most distinct are determinatives such as the, a, my, some, this, adjectives as pre-head modifiers, and restrictive relative clauses" Huddleston 1988, p. 85). Nouns can take determiners such as the, which, a, every, my, three. They can also take adjectives as pre-head modifiers (young girls, blue jeans) and relative clauses (people who sing). Nouns do not take objects (I dislike it/*my dislike it), as they cannot assign case.

c. Inflection: The contrasts of number, singular and plural, can be entered by adding inflections such as (camel vs camels) (Huddleston 2002:326; Huddleston 1988:84).

The most general of the three properties is the first one, since all nouns function as head in NP structure. Properties that include (b) and (c) characterize only central nouns, "with marginal members differing from them in varying degrees" (Huddleston 1984, p.227).

\section{Subclasses of nouns}

Nouns can be divided into several distinguishable subclasses, some of which will be introduced in the following section; at this point it will be sufficient to distinguish just three major subclasses as:

1. Common nouns: boy, rabbit, dress, etc..

2. Proper nouns: Azhar, Ahmad, Brazil, etc.

3. Pronouns: we, who, everybody, etc..

Proper nouns commonly function as the head of NP. They also serve as proper names. The difference between proper nouns and proper names is significant since, although a proper name will consist of a proper noun in the most basic cases, like Omar or Scotland, it may be structurally more complex. The University of Kuwait is a proper name, although it is not a proper noun. 
Proper nouns, such as Omar and Scotland, which can stand alone as proper names, are the most central type of proper nouns, and this is what sets them apart from common nouns. Those central proper nouns, however, are not restricted to functioning as the head of proper name NP (Huddleston, 1984:229-230).

\section{Countability; Plurality (Count, Non-count, and Proper Nouns)}

For both grammatical and semantics reasons, nouns are necessarily divided into subclasses. Taking four nouns, Mohammad, house, furniture and stone, this can be clearly demonstrated when we consider to what extent it is possible for each of these nouns to appear as head of the noun phrase, functioning as object in the sentence $I$ saw...; (a) with no determiner; (b) with the use of (the) as a determiner, (c) and, (d) some; (e) in the plural. The outcome of this test can be illustrated in Table (1):

Table 1. Test table for noun classes

\begin{tabular}{lllll} 
& 1 & 2 & 3 & $2+3$ \\
\cline { 2 - 4 } & Proper noun & + Count & - Count & $+/$ - Count \\
\hline a. & Mohammad & *house & furniture & stone \\
\hline b. & $*$ the Mohammad & The house & The furniture & The stone \\
\hline c. & $*$ a Mohammad & A house & $*$ a furniture & A stone \\
\hline d. & $*$ some Mohammad & *some house & Some furniture & Some stone \\
\hline e. & $*$ Mohammads & houses & $*$ furnitures & stones \\
\hline
\end{tabular}

Quirk (1985:246)

Proper Nouns are "nouns that belong to a grammatically distinct class of nouns which characteristically function as the head of NPs, serving as proper names" (Huddleston 1988, p. 96). These kind of nouns behave in the same way as the noun Mohammad (proper name) does, while the other nouns in the table are classified as Common Nouns. There are differences between Proper Nouns and Proper Names as I have previously mentioned, but I will not further discuss these differences, as the main focus of this paper is the difference between Count Nouns and Non-count Nouns. Count and Mass nouns do differ considerably from each other. Count Nouns behave like house in column (2) and denote individual, countable entities. Non-count Nouns, by contrast, follow the pattern of column (3) and denote an undifferentiated mass or continuum. Some nouns can be either count or non-count, as represented by those in column ( 2 +3) (e.g. brick, cake, paper, stone),

A noun like stone, for example, can be viewed as the non-count material (1), or it can constitute the countable object (2):

(1) The castle is built of stone

(2) The Romans used stones to build the castle.

Grammatically speaking, the last column can be classified in two ways:

a."A lexical class of noun combining the characteristics of count nouns and non-count nouns.

b. Two separate items, one count and the other non-count" (Quirk 1985, p.247).

Since there is little difference between the count and non-count uses for nouns like stone and cake in the former mode of analysis, such nouns are termed 'dual-class membership' (Quirk, 1985:247).

Nouns may seem superficially alike in terms of article usage, for example in the sentences I like makeup and I like Mohammad. Makeup has 'zero article', the label given to common nouns which have article contrast, while Mohammad has 'no article'.

\section{I like makeup.}

I think the makeup is too expensive.

In certain grammatical environments like the Mohammad I mean is handsome, proper nouns can have a determiner, but in most environments, they have no article contrast, and therefore are said to have 'no article' (Quirk, 1985:245).

\section{Noun Number and NP Number}

Both nouns and NPs are governed by the system of number (Huddleston 1988:87). In the case of nouns, the number is mainly an inflectional category: for example, rose and roses are the singular and plural forms of the lexeme rose, which is unspecified or neutral as to number. With regard to NPs, the noun functioning as head generally determines the number, although this is not necessarily so (Huddleston, 1984:239). 


\subsection{Determiner-head-co-occurrence restrictions of number}

While some determiners such as the, my, which, etc. can occur with either a singular or plural head, the rose or the roses, others are restricted to occurrences with only a singular or with a plural (Huddleston, 1984:240).

\section{a) Determiners that require a singular head}

"Another, this, that, either, neither, each, much, less, a little, every, little, one; (e.g. another baby/*babies has been born).

\section{b) Determiners that require a plural head}

Both, we, these, those, us, a few, many, several, few, fewer, two, three (etc.), (a) dozen (e.g. both windows $/{ }^{*}$ window smashed )" (Huddleston 1984, p. 240).

\subsection{Subject-verb agreement}

Co-occurrence restrictions involving agreement - number in combination with person - exist between subject and tensed verb, meaning that when the subject is $3^{\text {rd }}$ person singular, then the verb must reflect this. Likewise, if the subject is not $3^{\text {rd }}$ person singular, then this must be reflected by the verb; My brother lives/*live in Kuwait vs My brothers live/ *lives in Kuwait. "The agreement involves number in combination with person, and person is not an inflectional category in nouns" (Huddleston 1984, p.241).

\subsubsection{The basic exceptions to the basic agreement rule}

\subsubsection{Collectives}

Singular collective nouns, which denote a set or group of separate members, are an exception to the basic agreement rule as both forms of the verb might be used: The committee has/have accepted my application. The agreement rule is breached because the present tense form have lacks the singular property found in the subject. Rather than focusing on the set as a unit, the focus is on the individual members. Agreement is needed if the predicate applies to the set as a unit; The committee consists/*consist of three people.

With collective nouns, breach of agreement has the opposite effect of collectivizing. Elephant is not a collective noun (an elephant can only denote a single animal). Attention is shifted away from the members of the plurality by the syntactic construction of collectivizing in a plural NP. As a collective noun, committee denotes a set in the singular whereby the attention is shifted towards the individual members by the syntactic construction of discord.

The lexical factor, be it a collective or non-collective noun, outweighs the syntactic factor. The committee have is, therefore, more unified than three elephant, though less unified than the committee has. With collectives, discord is also related to the aspect of 'number-transparency'.

In an example such as The board of governors have decided not to make any further appointments, there are two alternatives:

1. "have is in discord with the 3rd person singular subject headed by collective board;

2. board is number-transparent, with governors determining the number of the board of governors as plural" (Huddleston, 1984, p.240).

\subsubsection{Quantities}

When the plural NP is interpreted as denoting a single quantity or amount, as opposed to a plurality of individuals, the opposite type of discord occurs; $3^{\text {rd }}$ person singular verb with $3^{\text {rd }}$ person plural NP as subject:

i Three mistakes in one paragraph is ridiculous.

ii Five inches is long enough.

iii Two pounds of sugar is the same weight as two pounds of feathers.

Whether or not are would be an acceptable alternative to is is uncertain. In (iii), for example, are seems better suited than $i s$ in (ii) (Huddleston, 1984:242).

\subsubsection{Proper names}

The verb form will be $3^{\text {rd }}$ person singular in the event of a plural NP being used as the name of a single entity: The Three Stooges is/*are my favorite movie.

The form of the verb is determined by the semantic predicate "be my favorite movie", as it refers to something singular, a movie.

In the case of a proper name being a collective such as The United Arab Emirates, point (c) will feed into point (a). As a plural NP naming a singular nation, it takes a $3^{\text {rd }}$ person singular verb similar to a singular proper name such as Kuwait. When applied to sporting teams representing a county, the names of countries can be used as collectives and take the general form of the present tense: The United Arab Emirates/ Kuwait have a lead of ten points to one.

In order to express different kinds of meaning, the choice of verb-form, $3^{\text {rd }}$ person singular or the general present tense, is significant. While grammar allows for a breach of agreement under certain conditions, the main determining factor is the syntactic number of the subject (Huddleston, 1984:243). 


\subsubsection{Lexically inherent number in nouns}

There is no inflectional contrast in a small number of nouns, and the number property, whether single or plural, is inherent in the noun lexeme itself. For example:

a) Nouns that are inherently singular

1. equipment, homework, knowledge, music, wetness

2. linguistics, mathematics, news

Although some non-count nouns may be pluralized, particularly those denoting natural phenomena; Let's lay on the sand/sands (Quirk, 1985:299), non-count nouns are singular and invariable (Huddleston, 1984:243-244).

\section{b) Nouns that are inherently plural}

1. clothes, earnings, greens (green vegetables), scissors, remains, etc.

2. cattle, people, police, etc.

The examples in the first category (a) are divided into two sets; in (1) they are straightforward, while those in (a2) appear to be plurals when they are actually singular. We say This news is hear breaking not *These news are heart breaking. In this case, there is no breach of agreement such as in The mistakes is too many. The verb behaves as syntactically singular, unlike one that agrees and/or is restricted to certain kinds of construction. They are not syntactic plurals.

The nouns in (b) are non-inflected plural. Cattle, for example, is a syntactically plural form, though it is not inflected for plural, and is not morphosyntactic; These cattle are healthy/*This cattle is healthy. Similarly, for police and people. People were rushing in the hospital, is lexically plural, and They are people of great morals, is the singular form of a regular collective noun with peoples as its counterpart; The Arabic-speaking peoples (Huddleston, 1984:243-44).

\subsubsection{How Inherently Plural Nouns are Classified in Terms of (+/- Count); Countability and boundedness}

Noun lexemes lacking singular or plural forms cannot enter into construction with determiners requiring those forms in head position; The noun surroundings, which is inherently plural, cannot combine with every, nor can phonetics, which is inherently singular, combine with many. Co-occurrence restrictions exist between determiners and nouns that cannot be explained in relation to simple contrasts between singular and plural; *two clothes, *another equipments. Rather, nouns must be classified in terms of degree of 'countability'. Uncountable nouns such as equipment, are incompatible with any of the following determiners:

i. The cardinal numerals one, two, etc.;

ii. Other numerically quantifying expressions both, a dozen, etc;

iii. Quantifiers like many, several, few;

iv. a, another, each, every, either, which take singular heads.

(Huddleston 1984:245)

There are by comparison fully countable nouns like horse, which can combine with any of these determiners. The intermediate classes are:

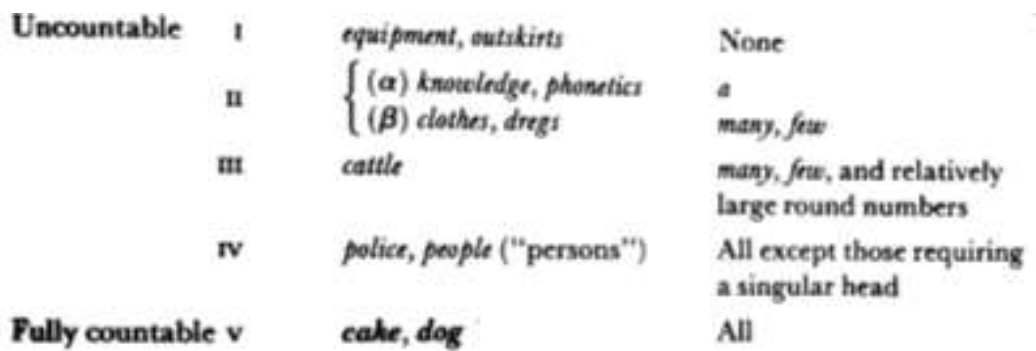

Taken from Huddleston (1984:245)

The extent to which a noun combines with different types of determiners reflects its countability. The interpretation of actual instance of nouns, however, must be taken into consideration. For example, the noun cake is interpreted differently in I'd like another cake and You shouldn't have eaten so much cake. The concept of 'boundedness' is the difference. A bounded or 'individuated' interpretation is denoted by the noun cake in another cake. It has an unbounded interpretation in the phrase so much cake as it is perceived as a unit. While Cake is a 'concrete' noun, the same distinction can be found in 'abstract' nouns. Countability is the main factor in boundedness. There are three factors to be considered when interpreting whether the noun is considered as mass or individuated. These factors being the number (singular/plural), the determiner, and also the noun itself. An uncountable noun cannot therefore bear an individuated 
interpretation. For example, with equipment, if one or more individual units are referred to, such expressions as a piece of equipment must be used. It consists of a mass NP embedded inside an individuated NP (Huddleston 1984:246).

\section{Reclassification}

It is possible to shift nouns from one class to another through conversation. A non-count noun like cheese can be 'reclassified' as a count noun. This involves a semantic shift to denote quality partition 'kind/type'. A noun like coffee, which is normally non-count (Would you like to order tea or coffee?), can be reclassified as a count noun. In this instance, it would mean an 'appropriate unit' (Two teas, please), meaning two cups of tea (Quirk 1985:248).

\section{Partitive construction}

\subsection{Partition in respect of quality}

Both count and non-count nouns can enter partitive constructions. These constructions denote a part of a whole and express both quality (a kind of paper) and quantity partitions (a piece of paper). Quality partition is expressed by a partitive count noun (kind, sort) followed by an of-phrase (Quirk, 1985:249).

\subsection{Partition in respect of quantity}

a. Non-count nouns:

Quantity and countability may be expressed by means of certain 'General Partitive Nouns' such as piece, bit, item; a piece of cake, two pieces of cake. In addition to some general partitives, there are other restricted 'Typical Partitives'. These form expressions with specific non-count nouns; a bar of chocolate/ soap/ gold (Quirk, 1985:250).

b. Plural count nouns:

With plural count nouns, a general partitive such as a piece of cannot be used.

c. Singular count nouns:

Partition can be used to refer to singular count nouns; a piece of loaf, a branch of a tree.

\subsection{Measure partitive nouns}

These nouns relate to precise quantities which might denote length, area, volume or weight; a foot of copper, an acre of land, a kilo of grapes. Measure partitive nouns can be either singular or plural; one gallon of water, two gallons of water. However, the second noun must be plural if it is countable; one kilo of grapes, two kilos of *grape (Quirk 1985:250).

\section{Nouns as Attributive Modifiers}

In traditional school grammar, nouns tends to be analyzed as adjectives; A government inquiry, Student performance, and The Caroline factor. This formulation causes confusion between categories and functions, whereby they are not nouns used solely as adjectives, but also as attributive modifiers. Most nouns can appear in this function, even proper nouns such as London and Clinton. Pronouns are the exception. These nouns can all appear as head of an NP in subject or object function. An attributive modifier is not considered a noun unless it has the same meaning as head of a NP.

As attributive nouns do not take very, too or more as a modifier, they do not qualify as adjectives. They do not meet the grading and adverbial dependents' criteria as they do not take adverbs as modifiers to the extent that they accept prehead modifiers. What the modifiers do is that they modify nouns functioning as head in NP structure.

In an example such as the federal government, a federal government inquiry, the adjective federal is the modifiers of government. Government does not take federal as an adverb; * federally government inquiry. The modifier is often another noun, as in biology student performance. "This difference in the category of modifiers applies equally in cases where there is homonymy between adjective and noun" (Huddleston 2002, p.537).

\section{Conclusion}

Nouns are classified into different classes and subclasses, as I have briefly mentioned in section 3.0. Countability and 'number' play an import role in the syntax of the English Language, as count nouns and non-count nouns behave differently in sentences. The difference between count and non-count nous have been discussed in section 4.0 and the discussion is carried out throughout this paper. This includes the subject-verb agreement, some exceptions to this agreement rule, lexically inherent number in nouns and partitive construction of count and non-count nouns. In the last section of this paper, I talked about nouns as attributive modifier, and gave some examples to illustrate my discussion.

\section{References}

Huddleston, R. (1984). Introduction to the Grammar Of English. UK: Cambridge University Press.

Huddleston, R. (1988). English Grammar: An Outline. UK: Cambridge University Press.

Huddleston, R. (2002). The Cambridge Grammar of the English Language. UK: Cambridge University Press.

Powell, D., Walker, E., \& Elsworth, S. (1989). Grammar Practice for Upper Intermediate Students. Pearson: Longman.

Quirk, R. (1985). A Comprehensive Grammar of the English Language. UK: Longman Group.

Robbins, L. M. (2007). Grammar and Style at Your Fingertips. USA: ALPHA BOOKS: Penguin Group.

Thomson, J., Martinet, A. V. (1986). A Practical English Grammar. Oxford: Oxford University Press. 